\title{
Gitelman syndrome disclosed by calcium pyrophosphate deposition disease: early diagnosis by ultrasonographic study
}

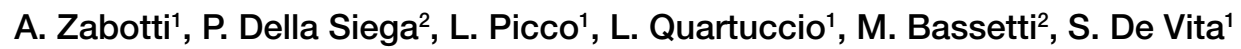 \\ ${ }^{1}$ Department of Medical and Biological Sciences, Rheumatology Clinic; \\ 2Infectious Disease Clinic, Santa Maria della Misericordia University-Hospital, Udine, Italy
}

\begin{abstract}
SUMMARY
Gitelman's syndrome is a rare autosomal-recessive tubular disorder characterized by hypomagnesemia and hypocalciuria associated to hypokalemia. The clinical spectrum is wide and usually characterized by chronic fatigue, cramps, muscle weakness and paresthesiae. We describe a case of a 43 year-old male patient with early onset of knee arthritis and no other symptoms. Ultrasound revealed diffuse and confluent hyperechoic deposits in cartilage, fibrocartilage of the menisci and synovium and calcium pyrophosphate crystals were observed in the synovial fluid of the knee. The concomitant presence of hypomagnesemia, hypocalciuria and hypokalemia made clear the diagnosis of Gitelman's syndrome associated with chondrocalcinosis.
\end{abstract}

Key words: Gitelman syndrome; calcium pyrophosphate deposition disease.

Reumatismo, 2016; 68 (1): 53-55

43-year old man presented at Early

Arthritis Clinic for recent onset of a mild swelling of the right knee, the remainder of the examination was normal and blood testing for inflammation and autoimmunity (i.e., erythrocyte sedimentation rate, C-reactive protein, rheumatoid factor, anti citrullinated peptide antibodies and antinuclear antibodies) were negative. Ultrasonography (US) of the involved knee revealed synovial hypertrophy with mild effusion and diffuse and confluent hyperechoic deposits within the femoral cartilage layer (A-B); other hyperechogenic deposits were highlighted in the menisci as well $(\mathrm{C})$.

The ultrasonographic appearance with hyperechoic deposits in the centre of the anhecoic hyaline femoral cartilage and the calcific deposits in meniscal fibrocartilage supported the suspicion of calcium pyrophosphate deposition disease (CPPD) (1-3). US pointed out a sub-clinic involvement of others joints, i.e. the left knee, ankles and metatarsal-phalangeal joints, characterized by the presence of calcifications of the articular cartilage, synovium, periarticular tissue and tendon (E-F), while the triangular fibrocartilage of the wrists, another typical site of crystal deposits (3), appeared normal. Knee arthrocentesis was carried out and calcium pyrophosphate crystals were observed in the synovial fluid by polarizing light microscopy. Since there was a polyarticular involvement with a diffuse and confluent hyperechoic deposits in cartilage, fibrocartilage of the menisci and synovium in a young adult, a metabolic disease or familial predisposition was suspected.

Laboratory analysis revealed hypomagnesemia $(0.64 \mathrm{mmol} / \mathrm{L}$, n.v. $0.72-1.25$ $\mathrm{mmol} / \mathrm{L})$, hypokalemia $(3.05 \mathrm{mmol} / \mathrm{L}$, n.v. 3.5-5.1 $\mathrm{mmol} / \mathrm{L}$ ) and hypocalciuria (0.40 mmol/24 h, n.v. $2.5-7.5 \mathrm{mmol} / \mathrm{L})$ and therefore a renal tubulopathy (Gitelman's disease) was suspected. The genetic analysis of gene $S L C 12 A 3$, showing two different double mutations, confirmed the diagnosis. A treatment with potassium and magnesium supplementation was started. Gitelman's syndrome is a rare autosomalrecessive tubular disorder, secondary to loss of function mutation in the $\mathrm{Na}-\mathrm{Cl}$ cotransporter located in the apical membrane of distal convoluted tubule. The hallmark of Gitelman's syndrome is hypomagnesemia and hypocalciuria associated to hy-
Indirizzo per la corrispondenza: Salvatore De Vita

Department of Medical and Biological Sciences

Rheumatology Clinic

Santa Maria della Misericordia

University-Hospital

P.le Santa Maria della Misericordia 15

33100 Udine

E-mail: devita.salvatore@aoud.sanita.fvg.it 



E


Figure 1 - Ultrasonographic examination of the femoral cartilage on longitudinal $(A)$ and transversal view $(B)$ with the right knee maximally flexed. In $A$ and $B$ ultrasonography examination reveals the hyperechoic deposits in the center of the anechoic hyaline femoral cartilage, due to diffuse deposition of calcium pyrophosphate crystals. Meniscal calcification of the right knee as it appears on ultrasonography (C) and corresponding radiograph (D) (white arrow). Transversal view of the right Achilles tendon (E) with anisotropic artefact and clear evidence of crystal deposits (curved arrow). $(\mathrm{F})$ Synovial hypertrophy with small crystal aggregates (curved arrow) in the second metatarsophalangeal joint of the right foot. In $(G)$ and $(H)$ radiographs of the left knee, clinically silent, with calcified enthesopathy of the quadriceps tendon $(\mathrm{G})$ and femoral cartilage calcification $(\mathrm{H})$. 
pokalemia. The clinical spectrum is wide and is characterized by chronic fatigue, cramps, muscle weakness and paresthesiae, nevertheless in our report none of these symptoms was present and knee arthritis was the only presenting symptom. Some authors have described an association between CPPD and Gitelman's syndrome (4-6). Probably, a key role is played by hypomagnesaemia because magnesium is a necessary cofactor for pyrophosphatases, a group of enzymes that increase the solubility of calcium pyrophosphate crystals.

This case report highlights the ultrasonographic features in different joints of a patient suffering from CPPD associated with Gitelman's syndrome.

The widespread calcifications are the main and well documented imaging features in such condition, but are usually shown and described on X-ray $(5,7)$.

Nevertheless, as US will be increasingly important to differentiate early arthritis (8), documenting ultrasonographic features of a rare disease, such as CPPD in Gitelman's syndrome, is worthwhile. In conclusion, this case report confirms the importance of US in the differential diagnosis of early arthritides.

Furthermore, from a clinical point of view, rheumatologists should pay attention to metabolic disease when widespread calcifications of the cartilage, synovium, tendon and periarticular tissue are detected.

\section{REFERENCES}

1. Filippou G, Adinolfi A, Bozios P, et al. Do not hallow until you are out of the wood! Ultrasonographic detection of CPP crystal deposits in menisci: facts and pitfalls. Sci World J. 2013; 2013: 1-6.

2. Grassi W, Meenagh G, Pascual E, Filippucci E. "Crystal clear"-sonographic assessment of gout and calcium pyrophosphate deposition disease. Semin Arthritis Rheum. 2006; 36: 197-202.

3. Filippou G, Filippucci E, Tardella M, et al. Extent and distrubution of CPP deposits in patients affected by calcium pyrophosphate dihydrate deposition disease: an ultrasonographic study. Ann Rheum Dis. 2013; 72: 1836-9.

4. Gutierrez M, Silveri F, Bertolazzi C, et al. Gitelman syndrome, calcium pyrophosphate dihydrate deposition disease and crowned dens syndrome. A new association? Rheumatology (Oxford). 2010; 49: 610-3.

5. Volpe A, Caramaschi P, Thalheimer U, et al. Familiar association of Gitelman's syndrome and calcium pyrophosphate dihydrate crystal deposition disease - a case report. Rheumatology (Oxford). 2007; 46: 1506-8.

6. Gutierrez M, Silveri F, Bertolazzi C, et al. [Gitelman syndrome associated with chondrocalcinosis: description of two cases.] Reumatismo. 2010; 62: 60-4. [Article in Italian]

7. Cobeta-Garcia JC, Gascón A, Iglesias E, Estopiñán V. Chondrocalcinosis and Gitelman's syndrome. A new association? Ann Rheum Dis. 1998; 57: 748-9.

8. Zabotti A, Salvin S, Quartuccio L, De Vita S. Differentiation between early rheumatoid and early psoriatic arthritis by the ultrasonographic study of the synovio-entheseal complex of the small joints of the hands. Clin Exp Rheumatol. 2016; 34: 459-65. 